\title{
Structural Operational Semantics for Continuous State Probabilistic Processes ${ }^{\star}$
}

\author{
Giorgio Bacci and Marino Miculan \\ Dept. of Mathematics and Computer Science, University of Udine, Italy \\ \{giorgio.bacci, marino.miculan\}@uniud.it
}

\begin{abstract}
We consider the problem of modeling syntax and semantics of probabilistic processes with continuous states (e.g. with continuous data). Syntax and semantics of these systems can be defined as algebras and coalgebras of suitable endofunctors over Meas, the category of measurable spaces. In order to give a more concrete representation for these coalgebras, we present an SOS-like rule format which induces an abstract GSOS over Meas; this format is proved to yield a fully abstract universal semantics, for which behavioural equivalence is a congruence.

To this end, we solve several problems. In particular, the format has to specify how to compose the semantics of processes (which basically are continuous state Markov processes). This is achieved by defining a language of measure terms, i.e., expressions specifically designed for describing probabilistic measures. Thus, the transition relation associates processes with measure terms.

As an example application, we model a CCS-like calculus of processes placed in an Euclidean space. The approach we follow in this case can be readily adapted to other quantitative aspects, e.g. Quality of Service, physical and chemical parameters in biological systems, etc.
\end{abstract}

\section{Introduction}

Process algebras are widely used for compositional modeling of nondeterministic, communicating, mobile systems. Categorically, the syntax of processes is represented as the initial algebra of a signature functor, and their semantics as coalgebras of a suitable "behavioral" functor. According to the Structural Operational Semantics (SOS) paradigm [25], these coalgebras are described by means of labelled transition systems (LTSs), defined by induction on the syntactic structure of processes. In order to guarantee important properties about the resulting semantics, several formats of these SOS specifications have been studied. A well-known format is the so-called GSOS [8], which guarantees the bisimilarity to be a congruence (in most situations). Such a framework makes languages easier to understand, compare, and extend. In particular, a process algebra can be easily extended with new operators, without the need of timeconsuming and error-prone proofs of congruence results.

` Work supported by MIUR PRIN project 20088HXMYN, "SisteR". 
In recent years, this approach has been applied also to stochastic and probabilistic systems, due to their important applications to performance evaluation, systems biology, etc [1991815. Bartels [6] and Klin and Sassone 20] have investigated rule formats (called Probabilistic GSOS and Stochastic GSOS, respectively) which guarantee bisimilarity to be a congruence.

However, these formats still do not cover the case of continuous-state (probabilistic) systems, like calculi with spatial/geometric features introduced in last years 104]. In these models, the behaviour of the system may be influenced by continuous data, which therefore is part of the state of the system. Typical examples are spatial informations, such as the position of processes and where transitions take place; e.g., in wireless networks distance may affect data access, or in biological models diffusion alters the signaling pathways, etc.

As a running example, in this paper we introduce a simple yet paradigmatic calculus of agents living in the Euclidean plane $\mathbb{R}^{2}$, which we call FlatCCS1. The idea we aim to model is that the probability of communications between two agents depends on their distance (like, e.g., in wireless networks). To this end, FlatCCS extends CCS (without restriction) with a syntactic "frame" operator representing a process' displacement:

$$
p, q::=\text { nil }|\alpha \cdot p| p+q|p \| q|[p]_{z} \quad \alpha::=a|\bar{a}| \tau
$$

where $a$ ranges over actions, and $z$ over the plane $\mathbb{R}^{2}$. Intuitively, if $p$ is in position $z^{\prime} \in \mathbb{R}^{2}$, the process $[p]_{z}$ is in $z^{\prime}+z$. If no frame operator occurs, processes are assumed to be in the origin $(0,0)$. Thus, in $\left[p \|[q]_{(0,1)}\right]_{(1,0)}, p$ is (externally) seen to be in $(1,0)$ and $q$ in $(1,1)$.

As for the semantics for this calculus, here we assume that the communication probability decreases exponentially with the distance. Thus, we expect the process $a$. nil $\|[\bar{a} . \text { nil }]_{(r, 0)}$ (with $\left.r \in \mathbb{R}\right)$ to perform a $\tau$ (that is, an internal communication) evolving into nil $\|[\text { nil }]_{(r, 0)}$ with probability $e^{-|r|}$. The problem is how to specify this semantics.

In [6], labelled probabilistic transition systems are shown to be coalgebras $g: X \rightarrow\left(\mathcal{D}_{\omega}(X)+1\right)^{L}$, where $L$ is the set of labels, and $\mathcal{D}_{\omega}$ is the probability distribution functor over Set (which gives the set of discrete finite supported probability distributions over a given set). This behaviour functor leads to transitions of the form $p \stackrel{\alpha[r]}{\longrightarrow} q$. However, we cannot use this approach for calculi like FlatCCS, because processes form a continuous space. This means that the probability of reaching any state $q$ from $p$ may be zero, yet the probability of reaching a subset of states may be nonzero.

The notion of interest is no longer a (discrete) probability distribution, but a (continuous) probability measure. Categorically, this corresponds to move to the category Meas of measurable spaces and measurable functions, and to model the a system behaviour by a coalgebra $g: X \rightarrow \Delta(X)^{L}$, where $\Delta$ is the Giry functor associating to $X$ the set of probability measures over $X$, as advocated in \begin{tabular}{ll|ll}
14 & 16 & 24
\end{tabular} . This leads to transitions of the form $p \stackrel{\alpha}{\rightarrow} \mu$, where $\mu$ is a measure of the probabilistic distribution of the possible outcomes of $p$.

\footnotetext{
${ }^{1}$ Of course other variants can be considered, e.g. LineCCS, SpaceCCS, etc. 11.
} 
Semantics with a similar transition format have been considered already in [113 for dealing with specific equational stochastic systems; these papers consider also suitable behavioural equivalences which are proved to be congruences. However, differently from the case of discrete (probabilistic) processes, SOS specifications and results in [113 are rather ad hoc, not based on any general framework for operational descriptions.

In this paper we aim to cover this gap, introducing a new GSOS rule format for probabilistic systems over measurable spaces. We will prove that this format guarantees that the resulting probabilistic behavioural equivalence is a congruence. As an example application, we will provide the semantics of FlatCCS according to this format.

To this end, we plan to apply the bialgebraic framework introduced by Turi and Plotkin [29]. However, in order to port this approach to our setting we have to solve several technical issues, due to the fact that we are working in Meas and using the Giry functor $\Delta$. First, $\Delta$ does not preserve weak pullbacks [2330], hence we cannot prove that bisimilarity is transitive and that it coincides with behavioural equivalence. As a consequence, we focus on behavioural equivalence instead of bisimilarity.

Secondly, Meas is not known to be Cartesian closed; hence, most of the constructions which can be carried out on Set and other toposes cannot be ported easily to Meas. In particular, we cannot follow Bartels's approach for deriving a rule format from a distributive law [6].

Moreover, a "good" SOS rule format must be compositional, i.e., it has to define a system's behaviour in terms of those of its subsystems. In traditional GSOS format, this is reflected by the fact that the target of a transition is a process built from the components of the source process, and their corresponding semantics. In our settings, the target of a transition is not a process term, but a measure over a generic measurable space, which do not have any syntactic structure to play with. In order to circumvent this problem, we propose to use transitions of the form $p \stackrel{\alpha}{\rightarrow} \mu$ where $\mu$ is a measure term, that is, a syntactic expression intended to denote a measure. The syntax of these measure terms, and their interpretation as measures, is part of the operational specification: a specification is given by a set of rules together with a description of how measures must be combined. We will show that this specification format, which we call Measure GSOS specification format, is general enough to cover the motivating example (and others [113]). In particular, we show that any LTS specification in this format leads to a distributive law of type $S\left(\operatorname{Id} \times \Delta^{L}\right) \Rightarrow\left(\Delta T_{S}\right)^{L}$, where $S$ is the syntactic functor and $T_{S}$ the corresponding free monad. As a consequence, the induced behavioural equivalence is always a congruence.

Synopsis. In Section 2, we recall the coalgebraic presentation of continuous probabilistic systems in the category Meas using the Giry functor.

In Section 3 we describe how to define syntactic monads over Meas. A technical issue here is that polynomial functors (as those arising from syntactic signatures) are not known to preserve $\omega$-colimits in Meas. To circumvent this 
problem, we use a more general initial algebra construction, which requires mild conditions about the base category.

Then, in Section 4 we introduce the MGSOS specification format. Using the previous results, we show that an MGSOS specification corresponds to a distributive law of type $S\left(I d \times \Delta^{L}\right) \Rightarrow\left(\Delta T_{S}\right)^{L}$, and the induced behavioural equivalence is always a congruence. As an exemplification, all results of this section are applied to FlatCCS.

As mentioned above, a key feature of this format is the fact that target measures are described by means of a specific term language, which have to be interpreted as measures. In Section $[5$ we show how to construct these interpretation functions using a generalized induction proof principle.

Final remarks and conclusions are in Section 6.

Measure Theoretic Preliminaries. A $\sigma$-algebra over a set $X$ is a non-empty family $\Sigma_{X}$ of subsets of $X$ closed under complements and countable unions. The pair $\left(X, \Sigma_{X}\right)$ is called measurable space and the members of $\Sigma_{X}$ are its measurable sets. A family of generators $\mathcal{F}$ for $\Sigma_{X}$ is a family of subsets of $X$ such that the smallest $\sigma$-algebra containing $\mathcal{F}$ is $\Sigma_{X}$, denoted by $\sigma(\mathcal{F})=\Sigma_{X}$.

Let $\left(X, \Sigma_{X}\right),\left(Y, \Sigma_{Y}\right)$ be measurable spaces, a function $f: X \rightarrow Y$ is called measurable if $f^{-1}(E)=\{x \mid f(x) \in E\} \in \Sigma_{X}$, for all $E \in \Sigma_{Y}$ (notably, if $\Sigma_{Y}$ is generated by $\mathcal{F}$, it suffices to show that $f^{-1}(F) \in \Sigma_{X}$, for all $F \in \mathcal{F}$ only).

A (sub-)probability measure on $\left(X, \Sigma_{X}\right)$ is a function $\mu: \Sigma_{X} \rightarrow[0,1]$ such that $\mu(X)=1$ (resp. $\leq 1)$, and it is $\sigma$-additive, i.e. $\mu\left(\bigcup_{i \in I} E_{i}\right)=\sum_{i \in I} \mu\left(E_{i}\right)$ for all countable collections $\left\{E_{i}\right\}_{i \in I}$ of pairwise disjoint measurable sets in $\Sigma_{X}$.

The set of sub-probability measures over $\left(X, \Sigma_{X}\right)$ forms a measurable space, denoted by $\Delta X$, with $\sigma$-algebra generated by $\left\{\mathcal{B}_{p}(E) \mid p \in[0,1], E \in \Sigma_{X}\right\}$, where $\mathcal{B}_{p}(E)=\{\mu \mid \mu(E) \geq p\}$.

\section{Continuous Probabilistic Systems as Coalgebras}

The coalgebraic treatment of continuous probabilistic systems originates in the work of de Vink and Rutten [13. Probabilistic bisimulation of Larsen and Skou 22] for discrete systems have been compared to the coalgebraic notion of bisimuation and ported without too much efforts to the continuous setting. Then, continuous probabilistic systems have been investigated from a coalgebraic point of view by Desharnais, Panagaden, Danos et al. [142412, which provided the most of the work available on (labelled) Markov processes, together with Doberkat [16] on stochastic relations.

In this section we briefly recall labelled continuous probabilistic systems, i.e. generalized labelled Markov processes over measurable spaces of states, and some peculiarities about the category Meas (details can be found in [27]).

Definition 1. For a set $L$ of action labels, a L-labelled Markov process on a measurable space $\left(X, \Sigma_{X}\right)$ is a structure $\left(X,\left\{\tau_{\alpha}: X \times \Sigma_{X} \rightarrow[0,1]\right\}_{\alpha \in L}\right)$, where $X$ is the set of states and, for each $\alpha \in L, \tau_{\alpha}$ is a transition sub-probability 
function, that is, a function such that, for all $x \in X, \tau_{\alpha}(x, \cdot)$ is a sub-probability measure, and, for each fixed $E \in \Sigma_{X}, \tau_{\alpha}(\cdot, E)$ is a measurable function.

Intuitively, $\tau_{\alpha}(x, E)$ is interpreted as the probability of the system starting in state $x$ making a $\alpha$-transition into one of the states in $E$. The transition probability is a conditional probability: it gives the probability of the system being in one of the states of the set $E$ after the transition, provided that it was in the state $x$ before the transition.

In order to model Markov processes as coalgebras one needs a suitable category and a suitable behaviour functor. The most natural choice for a category is Meas, the category of measurable spaces and measurable functions, and as for the behaviour functor, the Giry functor $\Delta$ : Meas $\rightarrow$ Meas, acting on objects as $X \mapsto \Delta X$ and on morphisms $f: X \rightarrow Y$ by $(\Delta f)(\mu)=\mu \circ f^{-1}$. This functor, first introduced by Lawvere, takes part in a monad triple commonly known as the Giry monad [17, with unit given by the Dirac measure $\delta_{X}$, given by $\delta_{X}(x)(E)=1$ if $x \in E, \delta_{X}(x)(E)=0$ otherwise 2 .

\section{Proposition 2. L-labelled Markov processes are exactly the $\Delta^{L}$-coalgebras.}

For different reasons in most of the works on Markov processes different categories were considered. In [13, de Vink and Rutten used ultrametric spaces arguing that the main reason for doing so was reusing a theorem that guarantees existence of a final coalgebra for locally contractive functors. Another reason for avoiding Meas is that the Giry functor does not preserve weak-pullbacks [30], which would be desirable for bisimilarity to be well-behaved. For instance, Desharnais, Edelat et al. 14 moved to the categories of analytic spaces in order to construct semi-pullback, providing a way to show that bisimilarity is transitive.

In this work we remain in Meas and argue that for the coalgebraic treatment of Markov processes it suffices to work with general measurable spaces unless one prefers bisimilarity to behavioral equivalence (i.e., the relation given by pullbacks on final coalgebra homomorphims). It is worthwhile to recall that in general, behavioural equivalence does not coincide with bisimilarity unless the behavior functor preserves weak pullbacks. This choice was already discussed by several authors, who have observed that in the generalized probabilistic setting, behavioral equivalence is more sensible than bisimilarity [12] and have suggested the use of co-congruence (also called event bisimulation) instead of the standard bisimulation. Similar arguments have been already discussed with more generality by Kurz in his doctoral thesis [21], and by Staton [28] where four different notions of bisimulation were investigated.

We conclude recalling some results that will be used later in the paper.

The category Meas is complete and cocomplete: limits and colimits are obtained as in Set and endowed, respectively, with initial and final $\sigma$-algebra w.r.t. their cone and cocone maps (indeed, the forgetful functor $U$ : Meas $\rightarrow$ Set preserves both limits and colimits).

${ }^{2}$ In [17, Giry considers actual probability measures, but it will be convenient to work with sub-probabilities instead. All the results go through also in this extended case. 
Completeness and cocompleteness of Meas allows us to consider the class of polynomial endofunctors, that is, the smallest class of endofunctors containing the identity $I d$, the constant functor $\underline{M}$ for all measurable spaces $M$, and closed under binary product and coproduct.

Moss and Viglizzo 2330] showed that polynomial functors extended with the Giry functor have a final coalgebra. Therefore, for $L$ a finite set of action labels, we are allowed to safely adopt behavioural equivalence over $\Delta^{L}$-coalgebras, since $\Delta^{L} \cong \prod_{\alpha \in L} \Delta$ is a finite product, hence admits a final coalgebra.

\section{Syntactic Monads over Measurable Spaces}

A crucial component of the bialgebraic approach is the syntactical monad associated with the endofunctor induced by the term syntax. Given a functor $F: \mathcal{C} \rightarrow \mathcal{C}$, the free monad associated to $F$ is defined on an object $X$ by means of the initial algebra of the functor $Y \mapsto X+F Y$. However, the usual construction of initial algebras as colimits of (initial) $\omega$-sequences, due to Smyth and Plotkin [26], cannot be applied to polynomial endofunctors in Meas, because they are not known to preserve colimits of $\omega$-sequences. However, we can adopt a generalization of [26] due to Adámek et al. [2] (see also Barr [5]), which extends to arbitrary ordinals the definition of initial sequence of $F$, where $\mathcal{C}$ is assumed to have an initial object 0 and colimits along ordinal indexed diagrams.

The construction is well known but we recall it for sake of clarity. The initial sequence of $F$ is an ordinal-indexed sequence of objects $\left(A_{\beta}\right)_{\beta \in \text { Ord }}$ with arrows $\left(f_{\beta}^{\gamma}: A_{\gamma} \rightarrow A_{\beta}\right)_{\gamma \leq \beta}$, uniquely defined by the following conditions, for $\delta \leq \gamma \leq \beta$ :

IS-1. $A_{\beta+1}=F A_{\beta}$;

IS-2. $f_{\beta+1}^{\gamma+1}=F f_{\beta}^{\gamma}$;

IS-3. $f_{\beta}^{\beta}=i d_{A_{\beta}}$ :

IS-4. $f_{\beta}^{\gamma} \circ f_{\gamma}^{\delta}=f_{\beta}^{\delta}$;

IS-5. if $\beta$ is a limit ordinal, the cocone $\left(f_{\beta}^{\gamma}: A_{\gamma} \rightarrow A_{\beta}\right)_{\gamma<\beta}$ is a colimit.

The sequence is given by transfinite induction on $\alpha \in \operatorname{Ord}$, defining $A_{\alpha}$ and $f_{\alpha}^{\beta}: A_{\beta} \rightarrow A_{\alpha}$, for all $\beta \leq \alpha$, and checking at each stage that conditions (IS-1) to (IS-5) hold for the portion of sequence already defined.

First step: Let $\alpha=0$. The sequence begins with $A_{0}=0$ and $f_{0}^{0}=i d_{A_{0}}$.

Isolated step: Let $\alpha=\alpha^{\prime}+1$ and assume by inductive hypothesis that $A_{\alpha^{\prime}}$ and the arrows $f_{\alpha^{\prime}}^{\gamma}$ have been given and satisfy (IS-1) define $A_{\alpha}=F A_{\alpha^{\prime}}$, and the arrows $f_{\alpha}^{\beta}$ are defined by induction on $\beta \leq \alpha$. We distinguish three cases. If $\beta=\alpha$, then we define $f_{\alpha}^{\beta}=i d_{A_{\alpha}}$. If $\beta$ is a successor ordinal, say $\beta=\beta^{\prime}+1$, then we define $f_{\alpha}^{\beta}=F f_{\alpha^{\prime}}^{\beta^{\prime}}$. If $\beta$ is a limit ordinal, $\left(f_{\beta}^{\gamma}: A_{\gamma} \rightarrow A_{\beta}\right)_{\gamma<\beta}$ is a colimit, by (IS-5). By inductive hypothesis on $\beta$, we can consider $\left(f_{\alpha}^{\gamma}: A_{\gamma} \rightarrow A_{\alpha}\right)_{\gamma<\beta}$, which turns out to be a compatible cocone by (IS-2) and (IS-4). Now, we define $f_{\alpha}^{\beta}$ as the unique map factorizing the cocone.

Limit step: Let $\alpha$ be a limit ordinal. By inductive hypothesis we are given all arrows $f_{\beta}^{\gamma}$, for $\gamma \leq \beta<\alpha$, which, indeed, form a chain. We define $A_{\alpha}$ to be the colimit of this chain and $\left(f_{\alpha}^{\beta}: A_{\beta} \rightarrow A_{\alpha}\right)_{\beta<\alpha}$ are its injections. 
Although not explicitly shown, in each step of the above construction conditions (IS-1) (IS-5) hold (see [5] for a detailed proof).

This sequence is defined through all ordinals, thus its construction does not terminate. If it should ever happen that $f_{\alpha+1}^{\alpha}$ is an isomorphism, it is clear that $f_{\beta}^{\alpha}$ is an isomorphism, for all $\beta>\alpha$. In this case we say that the sequence stabilizes (or terminates) at $\alpha$. Note that, since $f_{\alpha+1}^{\alpha}$ is an isomorphism it has inverse $\left(f_{\alpha+1}^{\alpha}\right)^{-1}: F A_{\alpha} \rightarrow A_{\alpha}$, which defines the structure map of an $F$-algebra with carrier $A_{\alpha}$. For this particular algebra we have the following result.

Theorem 3 ([5]). Let $\mathcal{C}$ be a category with initial object and colimits along ordinal-indexed diagrams, and $F: \mathcal{C} \rightarrow \mathcal{C}$ be a functor. Suppose the initial sequence of $F$ stabilizes at $\alpha$, then $\left(A_{\alpha},\left(f_{\alpha+1}^{\alpha}\right)^{-1}\right)$ is an initial $F$-algebra.

In many situations it is difficult to determine the ordinal $\alpha$ at which the initial sequence stabilizes. However, if the category $\mathcal{C}$ admits a factorization system, say $(\mathcal{E}, \mathcal{M})$, and it is $\mathcal{E}$-cowell-powered, the following result can be used instead.

Theorem 4. Let $(\mathcal{E}, \mathcal{M})$ be a factorization system for a $\mathcal{E}$-cowell-powered category $\mathcal{C}$, and $F: \mathcal{C} \rightarrow \mathcal{C}$ be a functor that preserves $\mathcal{E}$-morphisms. Suppose the initial sequence of $F$ has arrow $f_{\alpha+1}^{\alpha}: A_{\alpha} \rightarrow A_{\alpha+1}$ in $\mathcal{E}$, for some ordinal $\alpha$, then an initial F-algebra exists.

Proof. An easy transfinite induction argument shows that $f_{\delta}^{\beta}: A_{\beta} \rightarrow A_{\delta}$ are in $\mathcal{E}$, for all $\alpha \leq \beta \leq \delta$. The base case is clear. The successor step holds since $F$ preserves $\mathcal{E}$-morphisms, and for the limit step, one uses that $\mathcal{E}$ is closed by transfinite compositions. Hence we have that $f_{\beta}^{\alpha}$ are all $\mathcal{E}$ quotients of $A_{\alpha}$, for all $\beta \geq \alpha$. The result follows by the fact that $\mathcal{C}$ is $\mathcal{E}$-cowell-powered, so the initial sequence of $F$ stabilizes for some $\alpha^{\prime} \leq \alpha$.

Remark 5. On Meas, there are many factorization systems that "lift" the factorization system on Set, e.g., quotients and injections form a factorization system, and so do surjections and subspace embeddings. In order to apply Theorem 4, however, we cannot choose the former factorization system. Indeed, polynomial functors in Meas do not preserve quotients (it is well known that binary products fail to do so), but they do preserve surjections.

Recall that polynomial functors in Set preserves colimits of $\omega$-sequences, hence the initial sequence of these functors stabilizes at $\omega$. Now, since the forgetful functor $U$ : Meas $\rightarrow$ Set "maps" polynomial functors $P$ in Meas to polynomial functors in Set, and reflects surjections, the arrow $f_{\omega+1}^{\omega}: A_{\omega} \rightarrow A_{\omega+1}$ in the initial sequence of $P$ is surjective. Therefore, by Theorem 4 , there exists an initial $P$-coalgebra.

Notably, the existence of initial $P$-algebras ensures that the forgetful functor $U^{P}: P$-Alg $\rightarrow$ Meas has left adjoint $F^{P} \dashv U^{P}$. Therefore, one can construct the freely generated monad associated with $P$, denoted by $\left(T_{P}, \eta^{p}, \mu^{p}\right)$. In the case $P$ corresponds to a syntactic signature, this monad formally defines the measurable spaces of terms built over $P$, with unit $\eta^{p}: I d \Rightarrow T_{P}$ defined as the insertionof-variables function, and multiplication $\mu^{p}: T_{P}^{2} \Rightarrow T_{P}$ as the application-intocontexts function. Measurable sets for this space are induced by the cocone injections to the colimit (formally, to its sub-object) of the $P$-initial sequence. 


\section{Measure GSOS Specification Format}

In this section we present a (very general) technique for constructing GSOS distributive laws of type $S\left(I d \times \Delta^{L}\right) \Rightarrow\left(\Delta T_{S}\right)^{L}$ via sets of inference rules for a fixed syntactic format. This gives rise to a universal semantics (both initial and final) which is fully abstract w.r.t. $\Delta^{L}$-behavioural equivalence [29]. Moreover, the proposed method solves the problem of defining probability measures over continuous state spaces syntactically. This is done by using as targets of the transition relation, terms built over a new syntax, specifically designed for describing sub-probability measures.

Let $\mathcal{S}$ and $\mathcal{M}$ be two signatures, respectively called the process and the measure terms signatures. Let $S, M:$ Meas $\rightarrow$ Meas be the two polynomial functors associated with those signatures, formally given by 3

$$
S=\coprod_{f \in \mathcal{S}} I d^{\operatorname{ar}(f)} \quad M=\coprod_{f \in \mathcal{M}} I d^{\operatorname{ar}(f)} .
$$

By Remark [5] both $S$ and $M$ have their associated freely generated monads, namely, $\left(T_{S}, \eta^{s}, \mu^{s}\right)$ and $\left(T_{M}, \eta^{m}, \mu^{m}\right)$. These monads define the measurable spaces of terms built over the signatures $S$ and $M$, respectively.

Definition 6. A MGSOS rule over the signatures $S$ (for processes) and $M$ (for measure terms), for a finite set $L$ of labels, is an expression of the form

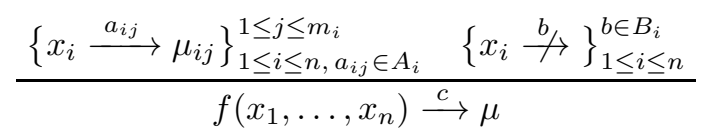

where

- $f \in S$ with $\operatorname{ar}(f)=n$;

$-\left\{x_{1}, \ldots, x_{n}\right\}$ and $\left\{\mu_{i j} \mid 1 \leq i \leq n, 1 \leq j \leq m_{i}\right\}$ are pairwise distinct variables;

- $A_{i} \cap B_{i}=\emptyset$ are disjoint subsets of labels in $L$, for all $1 \leq i \leq n$, and $c \in L$;

$-\mu$ is a measure term with variables in $\left\{x_{1}, \ldots, x_{n}\right\}$ and $\left\{\mu_{i j} \mid 1 \leq i \leq n, 1 \leq\right.$ $\left.j \leq m_{i}\right\}$.

Note that, differently from the standard GSOS rule format of [8], in the premises one is not allowed to use the same label twice for the same variable $x_{i}$; moreover, the source and target occurring in the conclusion have different signatures.

Together with each set of MGSOS rules we assume a natural transformation of type $T_{M} \Delta \Rightarrow \Delta T_{S}$, which interprets measure terms occurring in the target of the transitions as actual measures over the space of processes. This allows for a richer expressivity in the definition of a structural operational semantics.

Definition 7. A MGSOS specification system is a pair $(\mathcal{R}, \downarrow \cdot \downarrow)$, such that $\mathcal{R}$ is a set of image finite MGSOS rules, and $\downarrow \cdot \downarrow: T_{M} \Delta \Rightarrow \Delta T_{S}$ is a natural transformation, called measure terms evaluation.

${ }^{3}$ By an abuse of notation, in the rest of the paper we will often use the associated functor in place of its signature. 
Example 8. Let define the probabilistic semantics for FlatCCS by means of an MGSOS specification system $\left(\mathcal{R}^{\mathrm{fl}}, \downarrow \cdot \nu^{\mathrm{fl}}\right)$. The syntactic functors for FlatCCS processes and measure terms are given as follows, for a finite set $A$ of labels:

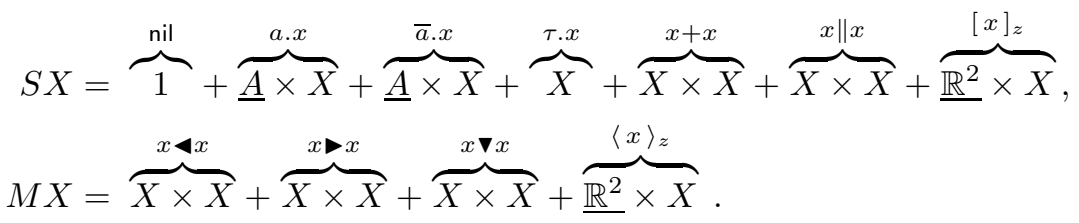

The set $\mathcal{R}^{\mathrm{fl}}$ of MGSOS rules gives the specification for a collective operational semantics in the form of a labelled transition system. A transition $p \stackrel{\alpha}{\longrightarrow} \mu$ has a process $p$ as source and a measure term $\mu$ as target, for $\alpha \in A \cup \bar{A} \cup\{\tau\}$.

$$
\begin{aligned}
& \underset{\alpha . x \stackrel{\alpha}{\longrightarrow} x}{\frac{x \stackrel{\alpha}{\longrightarrow} \mu}{x+x^{\prime} \stackrel{\alpha}{\longrightarrow} \mu}} \quad \frac{x^{\prime} \stackrel{\alpha}{\longrightarrow} \mu}{x+x^{\prime} \stackrel{\alpha}{\longrightarrow} \mu} \\
& \frac{x \stackrel{\alpha}{\longrightarrow} \mu}{[x]_{z} \stackrel{\alpha}{\longrightarrow}\langle\mu\rangle_{z}} \quad \frac{x \stackrel{\alpha}{\longrightarrow} \mu}{x \| x^{\prime} \stackrel{\alpha}{\longrightarrow} \mu \triangleleft x^{\prime}} \quad \frac{x^{\prime} \stackrel{\alpha}{\longrightarrow} \mu}{x \| x^{\prime} \stackrel{\alpha}{\longrightarrow} x>\mu}
\end{aligned}
$$

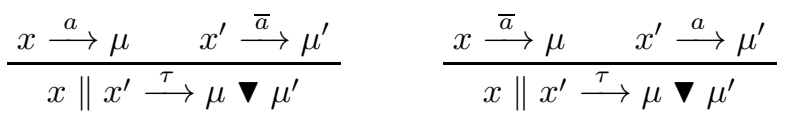

According to the rules above, $\mu \varangle \mu^{\prime}$ indicates that an action has been performed on the left hand side (dually in $\mu \mu^{\prime}$ ), $\mu \boldsymbol{\nabla} \mu^{\prime}$ denotes that the process succeeded in a synchronization, and $\langle\mu\rangle_{z}$ encodes the absolute position.

The semantics intuitively given in Section 1 is formalized by means of the measure term evaluation $\downarrow \cdot{ }^{\mathrm{fl}}: T_{M} \Delta \Rightarrow \Delta T_{S}$, defined, for $\mu, \mu^{\prime} \in T_{M} \Delta X$ and $\beta \in \Delta X$, as follows:4

$$
\begin{aligned}
\Delta \beta\rangle_{X}^{\mathrm{fl}} & =\beta \\
\left.\Delta \mu \triangleleft \mu^{\prime}\right\rangle_{X}^{\mathrm{fl}} & \left.\left.=(\Delta \mu\rangle_{X}^{\mathrm{fl}} \times \Delta \mu^{\prime}\right\rangle_{X}^{\mathrm{fl}}\right) \circ\left(\lambda\left(x, x^{\prime}\right) \cdot x \| x^{\prime}\right)^{-1} \\
\left.\Delta \mu \triangleright \mu^{\prime}\right\rangle_{X}^{\mathrm{fl}} & \left.\left.=(\Delta \mu\rangle_{X}^{\mathrm{fl}} \times \Delta \mu^{\prime}\right\rangle_{X}^{\mathrm{fl}}\right) \circ\left(\lambda\left(x, x^{\prime}\right) \cdot x \| x^{\prime}\right)^{-1} \\
\left\langle\mu \nabla \mu^{\prime}\right\rangle_{X}^{\mathrm{fl}} & \left.=\left(e^{-\left\|\operatorname{pos}(\mu)-\operatorname{pos}\left(\mu^{\prime}\right)\right\|} \cdot(\Delta \mu\rangle_{X}^{\mathrm{fl}} \times\left\langle\mu^{\prime}\right\rangle_{X}^{\mathrm{fl}}\right)\right) \circ\left(\lambda\left(x, x^{\prime}\right) \cdot x \| x^{\prime}\right)^{-1} \\
\left\langle\langle\mu\rangle_{z}\right\rangle_{X}^{\mathrm{fl}} & =\langle\mu\rangle_{X}^{\mathrm{fl}} \circ\left(\lambda x \cdot[x]_{z}\right)^{-1},
\end{aligned}
$$

where pos: $T_{M} \Delta X \rightarrow \mathbb{R}^{2}$ determines the position of an action by inspecting the syntactic structure of $\mu$, inductively defined by

$$
\begin{array}{ccr}
\operatorname{pos}(\beta)=(0,0) & \operatorname{pos}\left(\mu \varangle \mu^{\prime}\right)=\operatorname{pos}(\mu) & \operatorname{pos}\left(\mu \triangleright \mu^{\prime}\right)=\operatorname{pos}\left(\mu^{\prime}\right) \\
\operatorname{pos}\left(\mu \nabla \mu^{\prime}\right)=\frac{1}{2}\left(\operatorname{pos}(\mu)+\operatorname{pos}\left(\mu^{\prime}\right)\right) & \operatorname{pos}\left(\langle\mu\rangle_{z}\right)=z+\operatorname{pos}(\mu) .
\end{array}
$$

Note that the use of a new syntax in the target of a transition is essential in order to recover the absolute position of the action that has been performed.

${ }^{4}$ Here, $\beta \times \beta^{\prime}$ denotes the measure product, and $(r \cdot \beta)(E):=r \beta(E)$, for $0 \leq r \leq 1$. 
Similarly to GSOS transition systems specifications, also MGSOS specification systems define a structural operational semantics, but in this particular case in the form of a labelled Markov process over the measurable space of process terms. Its definition can be summarized in two stages. First, an image finite labelled transition system $\left(T_{S} 0,\left\{\stackrel{\alpha}{\longrightarrow} \subseteq T_{S} 0 \times T_{M}\left(T_{S} 0\right)\right\}_{\alpha \in L}\right)$ is defined using the set of MGSOS derivation rules, then the associated Markov process is obtained evaluating measure terms to sub-probability measures. Formally, the associated $\Delta^{L}$-coalgebra $\gamma$ over $T_{S} 0$ is defined, for $\alpha \in L$ and $p \in T_{S} 0$, by

$$
\left.\gamma(p)(\alpha)=\oplus_{T_{S} 0}\left(\{\backslash \mu\rangle_{T_{S} 0} \mid p \stackrel{\alpha}{\rightarrow} \mu\right\}\right) .
$$

where, for a finite set of $U$ sub-probability measures over $X$, the we define $\oplus_{X}(U): \Sigma_{X} \rightarrow[0,1]$ by $\oplus_{X}(U)(E)=\sum_{\mu \in U} \mu(E) / \sum_{\mu \in U} \mu(X)$, which is easily seen to be a (sub-)probability measure over $X$, and will be called (weighted) sum of sub-probability measures in $U$.

The main advantage in using this two staged definition is that Markov processes are defined syntactically by structural induction on process terms.

Example 9 (Trilateration). Let us see how the specification system for FlatCCS given in Example 8 may be used to model the node positioning problem using a trilateration strategy (as in GPS, robot position detection, etc). In order to determine its position, an agent calculates the distances of three (or more) beacon reference stations (anchors), whose positions are known. These distances can be derived from the communication probability between the agents and the anchors. A simple FlatCCS system implementing this scenario is the following:

$$
\begin{aligned}
\text { Sys } & =(\text { Agent } \| \text { Anchs }) \\
\text { Agent } & =\left[\left(\overline{a_{1}} \| \overline{a_{2}}\right) \| \overline{a_{3}}\right]_{(x, y)}, \\
\text { Anchs } & =\left(\left[a_{1}\right]_{\left(x_{1}, y_{1}\right)} \|\left[a_{2}\right]_{\left(x_{2}, y_{2}\right)}\right) \|\left[a_{3}\right]_{\left(x_{3}, y_{3}\right)} .
\end{aligned}
$$

There are only three possible transitions of the form $S y s \stackrel{\tau}{\rightarrow} \mu_{i}$, namely:

$$
\begin{aligned}
& \mu_{1}=\left\langle\left(\text { nil } 4 \overline{a_{2}}\right) \triangleleft \overline{a_{3}}\right\rangle_{(x, y)} \boldsymbol{\nabla}\left(\left(\langle\text { nil }\rangle_{\left(x_{1}, y_{1}\right)} \triangleleft\left\langle a_{2}\right\rangle_{\left(x_{2}, y_{2}\right)}\right) \triangleleft\left\langle a_{3}\right\rangle_{\left(x_{3}, y_{3}\right)}\right), \\
& \mu_{2}=\left\langle\left(\overline{a_{1}}>\mathrm{nil}\right) \triangleleft \overline{a_{3}}\right\rangle_{(x, y)} \boldsymbol{\nabla}\left(\left(\left\langle a_{1}\right\rangle_{\left(x_{1}, y_{1}\right)} \downarrow\langle\mathrm{nil}\rangle_{\left(x_{2}, y_{2}\right)}\right) \triangleleft\left\langle a_{3}\right\rangle_{\left(x_{3}, y_{3}\right)}\right) \text {, } \\
& \mu_{3}=\left\langle\left(\overline{a_{1}} \| \overline{a_{2}}\right) \triangleright \operatorname{nil}_{(x, y)} \nabla\left(\left(\left[a_{1}\right]_{\left(x_{1}, y_{1}\right)} \|\left[a_{2}\right]_{\left(x_{2}, y_{2}\right)}\right)>\langle\text { nil }\rangle_{\left(x_{3}, y_{3}\right)}\right)\right. \text {, }
\end{aligned}
$$

from which we can get the distances $\Delta_{i}$ from each anchor node $\left[a_{i}\right]_{\left(x_{i}, y_{i}\right)}$. In order to do so, for $\vec{E}=\left(E, E_{1}, E_{2}, E_{3}\right)$ a quadruple of measurable sets in $\mathbb{R}^{2}$, define the following measurable sets in $T_{S} 0$,

$$
\begin{aligned}
& \Gamma_{1}(\vec{E})=\left\{\left[\left(\text { nil } \| \overline{a_{2}}\right) \| \overline{a_{3}}\right]_{e} \|\left(\left([\text { nil }]_{e_{1}} \|\left[a_{2}\right]_{e_{2}}\right) \|\left[a_{3}\right]_{e_{3}}\right) \mid e \in E, e_{i} \in E_{i}\right\}, \\
& \Gamma_{2}(\vec{E})=\left\{\left[\left(\overline{a_{1}} \| \text { nil }\right) \| \overline{a_{3}}\right]_{e} \|\left(\left(\left[a_{1}\right]_{e_{1}} \|[\text { nil }]_{e_{2}}\right) \|\left[a_{3}\right]_{e_{3}}\right) \mid e \in E, e_{i} \in E_{i}\right\}, \\
& \Gamma_{3}(\vec{E})=\left\{\left[\left(\overline{a_{1}} \| \overline{a_{2}}\right) \| \text { nil }\right]_{e} \|\left(\left(\left[a_{1}\right]_{e_{1}} \|\left[a_{2}\right]_{e_{2}}\right) \|[\text { nil }]_{e_{3}}\right) \mid e \in E, e_{i} \in E_{i}\right\} .
\end{aligned}
$$

Exploiting the definition of $\downarrow \cdot \vee_{T_{S} 0}^{\mathrm{fl}}$ in Example 8 we have $\left\langle\mu_{i}\right\rangle_{T_{S} 0}^{\mathrm{fl}}\left(\Gamma_{i}(\vec{E})\right)=e^{-\Delta_{i}}$ whenever $(x, y) \in E$ and $\left(x_{j}, y_{j}\right) \in E_{j}$, for $1 \leq j \leq 3$ (otherwise is 0 ). Now, the 
coordinates $x$ and $y$ of the agent can be determined as the intersection of three circles with center in $\left(x_{i}, y_{i}\right)$ and radius $\Delta_{i}$, whose solution is given by

$$
\begin{aligned}
& y=\frac{2\left(V_{1,2}\left(x_{2}-x_{3}\right)-V_{3,2}\left(x_{2}-x_{1}\right)\right)}{\left(y_{1}-y_{2}\right)\left(x_{2}-x_{3}\right)-\left(y_{3}-y_{2}\right)\left(x_{2}-x_{3}\right)} \\
& x=\frac{y\left(y_{1}-y_{2}\right)-2 V_{1,2}}{x_{2}-x_{3}}
\end{aligned}
$$

where $V_{i, j}=\left(x_{j}^{2}-x_{i}^{2}\right)+\left(y_{j}^{2}-y_{i}^{2}\right)+\left(\Delta_{i}^{2}-\Delta_{j}^{2}\right)$, for $1 \leq i, j \leq 3$. Note that, in a more general situation, the position $(x, y)$ can only be stochastically determined with precision depending on the chosen measurable sets to be tested.

The next theorem shows how MGSOS specification systems induce natural transformation of type $S\left(I d \times \Delta^{L}\right) \Rightarrow\left(\Delta T_{S}\right)^{L}$, i.e., abstract GSOS laws of [29].

Theorem 10. An MGSOS specification system $(\mathcal{R}, \downarrow \cdot \nu)$ over $S$ and $M$, and set of labels $L$, determines a natural transformation of type $S\left(I d \times \Delta^{L}\right) \Rightarrow\left(\Delta T_{S}\right)^{L}$.

Proof. For any space $X$, we define $\llbracket \mathcal{R} \rrbracket_{X}: S\left(X \times(\Delta X)^{L}\right) \rightarrow\left(\Delta T_{S} X\right)^{L}$ in two steps as the composite of two (set) functions:

$$
S\left(X \times(\Delta X)^{L}\right) \longrightarrow \nu_{X} \longrightarrow\left(\mathcal{P}_{\text {fin }} T_{M} \Delta X\right)^{L} \stackrel{\left(\oplus T_{S} \circ \mathcal{P}_{\text {fin }} \downarrow \cdot \downarrow\right)_{X}^{L}}{\longrightarrow}\left(\Delta T_{S} X\right)^{L},
$$

where, for every space $X, \nu_{X}$ is defined as follows: for all $t \in T_{M} \Delta X, f \in S$, $c \in L, x_{i} \in X$, and $\beta_{i} \in(\Delta X)^{L}$, put

$$
t \in \nu_{X}\left(f\left(\left(x_{1}, \beta_{1}\right), \ldots,\left(x_{n}, \beta_{n}\right)\right)\right)(c)
$$

if and only if there exists a (possible renamed) rule in $\mathcal{R}$ with conclusion of the form $f\left(x_{1}, \ldots, x_{n}\right) \stackrel{c}{\rightarrow} \mu$, such that $\beta_{i}(b)=\omega$, for $b \in B_{i}$, and $t=\left(T_{M} \sigma\right)(\mu)$ for a substitution map $\sigma$ such that $\sigma\left(x_{i}\right)=\delta_{x_{i}}$ and $\sigma\left(\mu_{i j}\right)=\beta_{i}\left(a_{i j}\right)$ (the indices $i, j$ follows the conventions of Definition [6).

Naturality of $\llbracket \mathcal{R} \rrbracket$ is proved separately for the two components. Since $\langle\cdot\rangle$ and $\oplus$ are natural, the second component is easily seen to be natural as well; whereas for naturality of $\nu$ one proceeds as in [29, Th. 1.1].

As for measurability of $\llbracket \mathcal{R} \rrbracket_{X}$, it suffices to check that $\llbracket \mathcal{R} \rrbracket_{X}^{-1}\left(U_{\alpha}[E]\right)$ is measurable in $S\left(X \times(\Delta X)^{L}\right)$, for $U_{\alpha}[E]=\left\{\beta^{\prime} \in\left(\Delta T_{S} X\right)^{L} \mid \beta^{\prime}(\alpha) \in E\right\}$, with $\alpha \in L$, and $E \in \Sigma_{\Delta T_{S} X}$.

${ }^{5}$ In general, this is a systems of three independent non-linear equations, hence it may have multiple or no real solutions. Here, each radius is determined by measuring the distance from the intersecting point, therefore it has always a unique solution. 
Let $f(\overline{x, \beta})$ be an abbreviation for $f\left(\left(x_{1}, \beta_{1}\right), \ldots,\left(x_{n}, \beta_{n}\right)\right) \in S\left(X \times(\Delta X)^{L}\right)$, then we have

$$
\begin{aligned}
\llbracket \mathcal{R} \rrbracket_{X}^{-1}\left(U_{\alpha}[E]\right) & =\left\{f(\overline{x, \beta}) \mid \llbracket \mathcal{R} \rrbracket_{X}(f(\overline{x, \beta})) \in U_{\alpha}[E]\right\} \\
& =\left\{f(\overline{x, \beta}) \mid\left(\left(\oplus T_{S} \circ \mathcal{P}_{\text {fin }} \downarrow \cdot \nu\right)^{L} \circ \nu\right)_{X}(f(\overline{x, \beta})) \in U_{\alpha}[E]\right\} \\
& =\left\{f(\overline{x, \beta}) \mid\left(\left(\oplus T_{S} \circ \mathcal{P}_{\text {fin }} \downarrow \cdot \nu\right)^{L} \circ \nu\right)_{X}(f(\overline{x, \beta}))(\alpha) \in E\right\} \\
& =\left\{f(\overline{x, \beta}) \mid\left(\oplus T_{S} \circ \mathcal{P}_{\text {fin }} \downarrow \cdot \nu \circ \nu^{\alpha}\right)_{X}(f(\overline{x, \beta})) \in E\right\}
\end{aligned}
$$

where $\nu_{X}^{\alpha} \triangleq \nu_{X}(\cdot)(\alpha)$ is the specialization of $\nu_{X}$ on a fixed $\alpha \in L$,

$$
\begin{aligned}
& =\left(\oplus T_{S} \circ \mathcal{P}_{\text {fin }} \downarrow \cdot \nu \circ \nu^{\alpha}\right)_{X}^{-1}(E) \\
& =\left(\nu_{X}^{\alpha}\right)^{-1} \circ\left(\oplus T_{S} \circ \mathcal{P}_{\text {fin }} \downarrow \cdot \nu\right)_{X}^{-1}(E)
\end{aligned}
$$

Now is easy to prove measurability. Since $\downarrow \cdot \nabla$ is measurable and sums and products of measurable functions is measurable, $\left(\oplus T_{S} \circ \mathcal{P}_{\mathrm{fin}} \downarrow \cdot \nu\right)_{X}^{-1}(E) \in \Sigma_{\mathcal{P}_{\mathrm{fin}} T_{M} \Delta X}$.

To prove measurability of $\nu_{X}^{\alpha}$ we need only to check that $\left(\nu_{X}^{\alpha}\right)^{-1}\left(\bigcup_{j=0}^{k}\left\{E_{j}\right\}\right)$ is a measurable set in $S\left(X \times(\Delta X)^{L}\right)$, for $E_{j} \in \Sigma_{T_{M} \Delta X}, 0 \leq j \leq k$.

$$
\begin{aligned}
\left(\nu_{X}^{\alpha}\right)^{-1}\left(\bigcup_{j=0}^{k}\left\{E_{j}\right\}\right) & =\left\{f(\overline{x, \beta}) \mid \nu_{X}^{\alpha}(f(\overline{x, \beta})) \in \bigcup_{j=0}^{k}\left\{E_{j}\right\}\right\} \\
& =\bigcup_{j=0}^{k}\left\{f(\overline{x, \beta}) \mid \nu_{X}^{\alpha}(f(\overline{x, \beta}))=E_{j}\right\}
\end{aligned}
$$

But $\nu_{X}^{\alpha}(f(\overline{x, \beta}))=E_{j}$ iff there exists some rule in $\mathcal{R}$ with conclusion of the form $f\left(x_{1}, \ldots, x_{n}\right) \stackrel{\alpha}{\rightarrow} \mu$ and $\left(T_{M} \sigma\right)(\mu) \in E_{j}$ (obviously, all the other conditions given above have to be satisfied too). By construction, $\sigma$ is defined by sums of Dirac measures $\delta_{X}$ and $e v_{a_{i j}}$, which are measurable. Therefore, $T_{M} \sigma$ is measurable, and as a consequence also $\nu_{X}^{\alpha}$ is measurable.

In the above proof, measure terms variables are interpreted as Dirac measures via the natural transformation $\delta: I d \Rightarrow \Delta$ (hence, measurable in each component). This together with the assumption that $\downarrow \cdot \downarrow: T_{M} \Delta \Rightarrow \Delta T_{S}$ is a natural transformation are crucial to prove measurability of $\llbracket \mathcal{R} \rrbracket$.

Example 11. For the MGSOS specification system given in Example 8 the construction in Theorem [10, for $x, x^{\prime} \in X$ and $\beta, \beta^{\prime} \in(\Delta X)^{L}$, gives

$$
\llbracket \text { nil } \rrbracket_{X}=\lambda \alpha . \omega
$$

$$
\llbracket \alpha . \rrbracket_{X}(x, \beta)=\lambda \alpha^{\prime} . \begin{cases}\left\langle\delta_{x}\right\rangle_{X}^{\mathrm{fl}} & \text { if } \alpha^{\prime}=\alpha \\ \omega & \text { otherwise }\end{cases}
$$

$(x, \beta) \llbracket+\rrbracket_{X}\left(x^{\prime}, \beta^{\prime}\right)=\lambda \alpha \cdot \Delta \beta(\alpha) \nu_{X}^{f l} \oplus\left\langle\beta^{\prime}(\alpha) \nu_{X}^{f l}\right.$

$(x, \beta) \llbracket \| \rrbracket_{X}\left(x^{\prime}, \beta^{\prime}\right)=\lambda \alpha \cdot \begin{cases}\left\langle\beta(\alpha) \triangleleft \delta_{x^{\prime}}\right\rangle_{X}^{\mathrm{fl}} \oplus\left\langle\delta_{x}>\beta^{\prime}(\alpha) \nu_{X}^{\mathrm{fl}}\right. & \text { if } \alpha \neq \tau \\ \bigoplus_{a \in A}\left\{\begin{array}{c}\left.\Delta \beta(\alpha) \triangleleft \delta_{x^{\prime}}\right|_{X} ^{\mathrm{fl}},\left\langle\delta_{x}-\beta^{\prime}(\alpha) \nu_{X}^{\mathrm{fl}},\right. \\ \left\langle\beta(a) \nabla \beta^{\prime}(\bar{a}) \nu_{X}^{\mathrm{fl}},\left\langle\beta(\bar{a}) \boldsymbol{\nabla} \beta^{\prime}(a)\right\rangle_{X}^{\mathrm{fl}}\right.\end{array}\right\} & \text { if } \alpha=\tau\end{cases}$ $\left.\llbracket[]_{z} \rrbracket_{X}(x, \beta)=\lambda \alpha^{\prime} \cdot \triangleleft \beta(\alpha)\right\rangle_{X}^{\mathrm{fl}}$ 
where $\omega$ is the null sub-probability measure assigning to each measurable set probability zero. Note that, since $A$ is finite, the last summand is well defined.

When the measurable space of variables $X$ is taken to be the space $T_{S} 0$ of ground measurable terms, the above definition gives the "canonical" initial denotational semantics for FlatCCS.

In [29], it is shown that distributive laws $\rho: \Sigma(I d \times B) \Rightarrow B T_{\Sigma}$, where $T_{\Sigma}$ is the free monad over $\Sigma$, for endofunctors $\Sigma$ and $B$ admitting initial $\Sigma$-algebra $\left(T_{\Sigma} 0, \alpha\right)$ and final $B$-coalgebra $(F, \omega)$, give rise to a unique $B$-coalgebra structure $\beta_{\rho}: T_{\Sigma} 0 \rightarrow B T_{\Sigma} 0$ such that $\left(T_{\Sigma} 0, \alpha, \beta_{\rho}\right)$ is the initial $\rho$-model (i.e., the initial $\rho$-bialgebra). Dually, there is a unique $\Sigma$-algebra structure $\alpha_{\rho}: \Sigma F \rightarrow F$ such that $\left(F, \alpha_{\rho}, \beta\right)$ is the final $\rho$-model. Thus, the following holds:

Theorem 12 (Adequacy 29]). Let $\rho$ be an abstract distributive GSOS law. The unique (both by initiality and finality) homomorphism from the initial to the final $\rho$-model is both the initial and final semantics for $\rho$.

Due to this result, the above is called universal semantics for $\rho$. Note, that two $B$ coalgebras have the same universal semantics if and only if they are behavioural equivalent, therefore $B$-behavioural equivalence is a $\Sigma$-congruence.

As for abstract GSOS laws of type $S\left(I d \times \Delta^{L}\right) \Rightarrow\left(\Delta T_{S}\right)^{L}$ we have the following result for behavioural equivalence on probabilistic processes on Meas.

Corollary 13. Behavioural equivalence on the $\Delta^{L}$-coalgebras over $T_{S} 0$ inductively induced by MGSOS specification systems over $S$ and $L$, is a $S$-congruence.

Proof. By Theorem 4 and Remark [5] every syntactic functor $S:$ Meas $\rightarrow$ Meas have initial algebra. As for the endofunctor $\Delta^{L}:$ Meas $\rightarrow$ Meas, a proof of the existence of the final coalgebra is given in 30 . The thesis follows combining Theorems 10 and 12 .

\section{$5 \quad$ Measure Terms Evaluations}

MGSOS specifications require the evaluations $\downarrow \cdot \lambda_{X}: T_{M} \Delta X \rightarrow \Delta T_{S} X$ to be both natural and measurable. This time consuming check is overcome in this section, where we provide a rather easy (and general) technique for defining natural measure terms evaluation functions.

This technique in based on a generalized induction proof principle, dual to the "coiterative proof principle" described in [6]. This method is then shown to work for the definition of measure terms evaluation for FlatCCS of Example 8 .

We start giving a recursion lemma dual to [6], which generalizes the standard induction proof principle by means of a simple distributive law $\lambda$.

Lemma 14. Let $S, B: \mathcal{C} \rightarrow \mathcal{C}$ be functors on a category with countable products, $(A, \alpha)$ be the initial $S$-algebra, and $\lambda: S B \Rightarrow B S$ be a (simple) distributive law. 
For any $S B$-algebra $(X, \varphi)$ there exists a unique $\lambda$-iterative arrow $f: A \rightarrow X$ induced by $\varphi$, such that the following diagrams commute
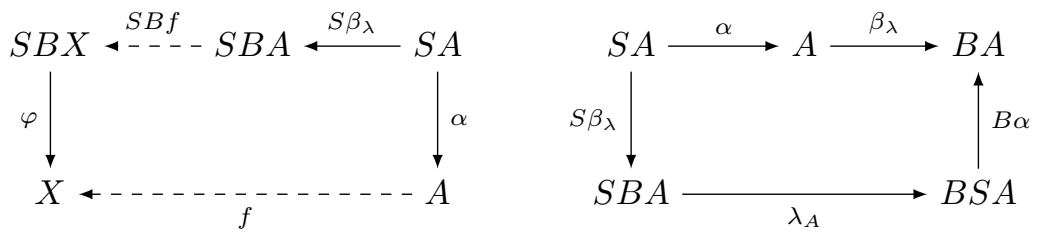

Proof. Dualize [6, Theorem 4.2.2].

We denote this induction proof principle by $\lambda$-iteration proof principle, and we call $f: A \rightarrow X$ as the $\lambda$-iterative arrow induced by $\varphi$. Note that, the diagram on the right is the initial $\lambda$-model, and in particular $\beta_{\lambda}$ is uniquely determined by (standard) induction on the $S$-algebra $\left(B A, B \alpha \circ \lambda_{A}\right)$.

The $\lambda$-iteration proof principle of Lemma 14 can be extended as a proof principle on the monad $T_{S}$ freely generated by $S$ as follows:

Proposition 15 (Structural $\lambda$-iteration). Let $S, B: \mathcal{C} \rightarrow \mathcal{C}$ be functors on a category with binary coproducts and countable products, $\left(T_{S}, \eta^{s}, \mu^{s}\right)$ be the free monad over $S, \lambda: S B \Rightarrow B S$ be a distributive law, and $\psi_{X}: S T_{S} X \rightarrow T_{S} X$ be the free $S$-algebra structure over $X$. For any $S B$-algebra $(Y, \varphi), B$-coalgebra $(X, k)$, and arrow $\phi: X \rightarrow Y$, there exists a unique arrow $f: T_{S} X \rightarrow Y$ such that the following diagrams commute
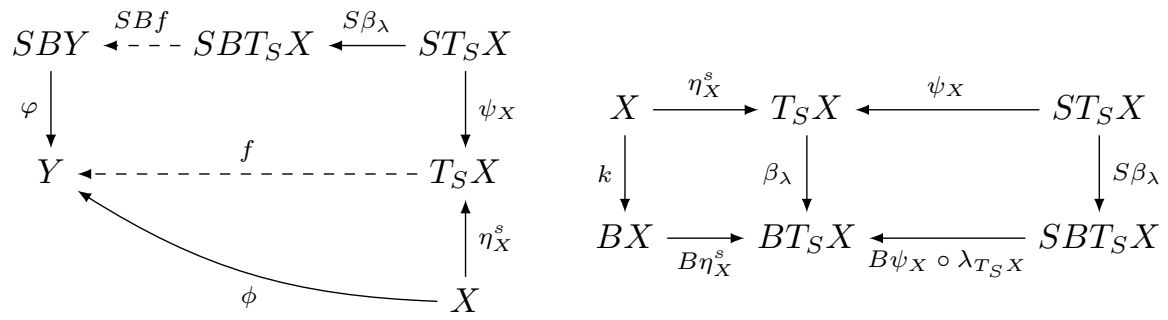

Proof. First, notice that $\beta_{\lambda}$ is the inductive extension of the $(\underline{X}+S)$-algebra structure on $B T_{S} X$ given by the copair $\left[B \eta_{X}^{s} \circ k, B \psi_{X} \circ \lambda_{T_{S} X}\right]$, along the initial $(\underline{X}+S)$-algebra structure $\left[\eta_{X}^{s}, \psi_{X}\right]$ on $T_{S} X$. Now, define the distributive law $\lambda^{\prime}:(\underline{X}+S) B \Rightarrow B(\underline{X}+S)$ as $\lambda_{Y}^{\prime}=\left[\operatorname{Bin}_{l}, B i n_{r}\right] \circ\left(k+\lambda_{Y}\right)$ (the proof of naturality is straightforward). By definition of $\lambda^{\prime}$ we have

$$
\left[B \eta_{X}^{s} \circ k, B \psi_{X} \circ \lambda_{T_{S} X}\right]=B\left[\eta_{X}^{s}, \psi_{X}\right] \circ \lambda_{T_{S} X}^{\prime}
$$

Therefore, by unicity of the inductive extension, $\left(T_{S} X, \psi_{X}, \beta_{\lambda}\right)$ turns out to be a $\lambda^{\prime}$-model on $T_{S} X$. This allows to apply Lemma 14 on the $(\underline{X}+S) B$-algebra structure $(Y,[\phi, \varphi])$ obtaining a unique $\lambda^{\prime}$-iterative arrow $f: T_{S} X \rightarrow Y$ making the required diagrams above commute. 
We denote this proof principle by structural $\lambda$-induction proof principle, and we say that $f$ is the $\lambda$-iterative extension of $\varphi$ along the (pair of) valuation functions $\phi$ and $k$. Note that, the diagram on the right define $\beta_{\lambda}$ as the structural inductive extension of $B \psi_{X} \circ \lambda_{T_{S} X}$ along the valuation function $B \eta_{X}^{s} \circ k$.

Proposition 15 can be turned into an induction proof principle on natural transformations in the following way:

Corollary 16. Let $S, B, F: \mathcal{C} \rightarrow \mathcal{C}$ be functors on a category with binary coproducts and countable products, $\left(T_{S}, \eta^{s}, \mu^{s}\right)$ be the free monad over $S, \lambda: S B \Rightarrow B S$ be a distributive law, and $\psi: S T_{S} \Rightarrow T_{S}$ be the natural transformation defined on any component $X$ as the free $S$-algebra structures over $X$. For any $\varphi: S B F \Rightarrow F, k: I d \Rightarrow B$, and $\phi: I d \Rightarrow F$, there exists a unique natural transformation $f: T_{S} \Rightarrow F$ such that the following (natural) diagrams commute
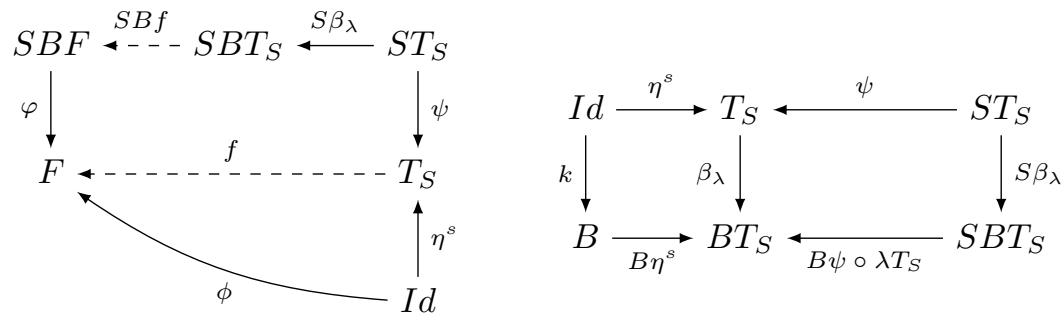

where $\beta_{\lambda}: T_{S} \Rightarrow B T_{S}$ is defined on any component $X$ as the unique inductive extension of $B \psi_{X} \circ \lambda_{T_{S} X}$ along the valuation function $B \eta_{X}^{s} \circ k_{X}$.

Proof. We first prove naturality of $\beta_{\lambda}$, i.e., that for any morphism $g: X \rightarrow Y$, $\left(\beta_{\lambda}\right)_{Y} \circ T_{S} g=B T_{S} g \circ\left(\beta_{\lambda}\right)_{X}$. The commuting diagrams

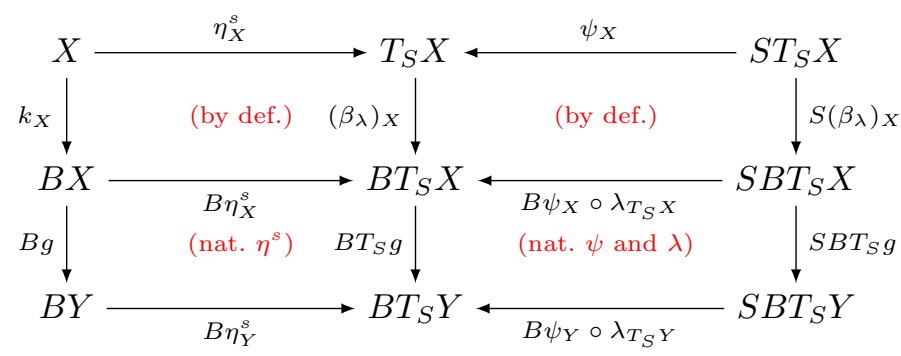

and

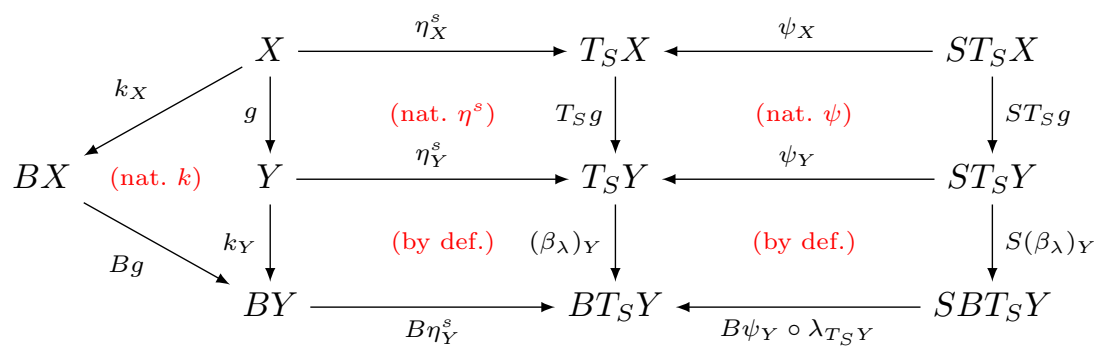


assert that $\left(\beta_{\lambda}\right)_{Y} \circ T_{S} g$ and $B T_{S} g \circ\left(\beta_{\lambda}\right)_{X}$ are both inductive extensions of $B \psi_{Y} \circ \lambda_{T_{S} Y}$ along $B \eta_{X}^{s} \circ B g \circ k_{X}$, hence they necessarily coincide.

Naturality of $f$ follows similarly by unicity of the $\lambda$-iterative extension. Indeed, both $f_{Y} \circ T g$ and $F g \circ f_{X}$ are $\lambda$-iterative extensions of $\varphi_{Y}$ along the pair of valuations $F g \circ \phi_{X}$ and $k_{X}$, as proved by the commutative diagrams

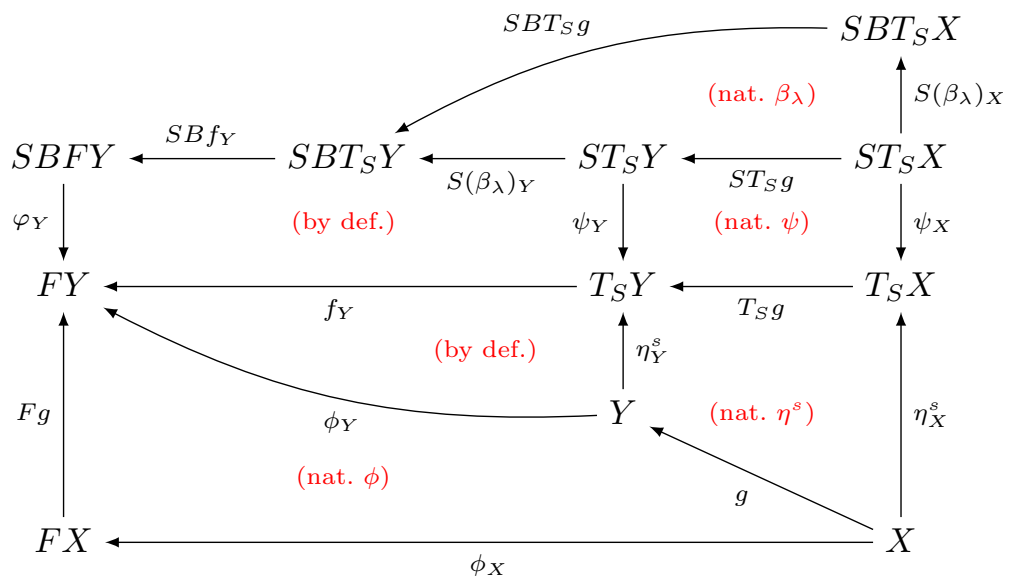

and

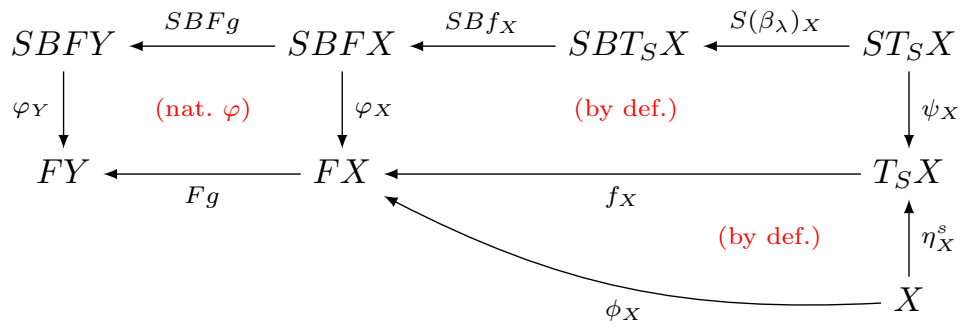

The above result turns out to be a useful tool to easily obtain measure evaluation functions satisfying the naturality and measurability requirements.

Example 17. Consider the measure terms evaluation function for FlatCCS given in Example8. Here we show how it arises as an instance of structural $\lambda$-iteration, for a suitable distributive law of type $\lambda: M\left(\underline{\mathbb{R}^{2}} \times I d\right) \Rightarrow\left(\underline{\mathbb{R}^{2}} \times I d\right) M$.

Let $\lambda: M\left(\underline{\mathbb{R}^{2}} \times I d\right) \Rightarrow\left(\underline{\mathbb{R}^{2}} \times I d\right) M$ be, for $x, x^{\prime} \in X$ and $z, z^{\prime} \in \mathbb{R}^{2}$,

$$
\begin{aligned}
\lambda_{X}\left((z, x) \triangleleft\left(z^{\prime}, x^{\prime}\right)\right) & =\left(z, x \bullet x^{\prime}\right) \\
\lambda_{X}\left((z, x) \triangleright\left(z^{\prime}, x^{\prime}\right)\right) & =\left(z^{\prime}, x \triangleright x^{\prime}\right) \\
\lambda_{X}\left((z, x) \boldsymbol{\nabla}\left(z^{\prime}, x^{\prime}\right)\right) & =\left(\frac{1}{2}\left(z+z^{\prime}\right), x \boldsymbol{\nabla} x^{\prime}\right) \\
\lambda_{X}\left(\langle(z, x)\rangle_{z^{\prime}}\right) & =\left(z+z^{\prime},\langle x\rangle z^{\prime}\right) .
\end{aligned}
$$


Then, define $k: I d \Rightarrow\left(\underline{\mathbb{R}^{2}} \times I d\right)$, and a $\varphi: M\left(\underline{\mathbb{R}^{2}} \times \Delta T_{S}\right) \Rightarrow \Delta T_{S}$ as follows, for $x \in X, z, z^{\prime} \in \mathbb{R}^{2}$, and $\beta, \beta^{\prime} \in \Delta T_{S} X$,

$$
\begin{aligned}
k_{X}(x) & =((0,0), x), \\
\varphi_{X}\left((z, \beta) \triangleleft\left(z^{\prime}, \beta^{\prime}\right)\right) & =\left(\beta \times \beta^{\prime}\right) \circ\left(\lambda\left(x, x^{\prime}\right) \cdot x \| x^{\prime}\right)^{-1} \\
\varphi_{X}\left((z, \beta) \triangleright\left(z^{\prime}, \beta^{\prime}\right)\right) & =\left(\beta \times \beta^{\prime}\right) \circ\left(\lambda\left(x, x^{\prime}\right) \cdot x \| x^{\prime}\right)^{-1} \\
\varphi_{X}\left((z, \beta) \mathbf{\nabla}\left(z^{\prime}, \beta^{\prime}\right)\right) & =\left(e^{-\left\|z-z^{\prime}\right\|} \cdot\left(\beta \times \beta^{\prime}\right)\right) \circ\left(\lambda\left(x, x^{\prime}\right) \cdot x \| x^{\prime}\right)^{-1} \\
\varphi_{X}\left(\langle(z, \beta)\rangle_{z^{\prime}}\right) & =\beta \circ\left(\lambda x \cdot[x]_{z^{\prime}}\right)^{-1} .
\end{aligned}
$$

These are easily seen to be natural and measurable. Now, applying the structural $\lambda$-iteration proof principle of Proposition 15 we obtain
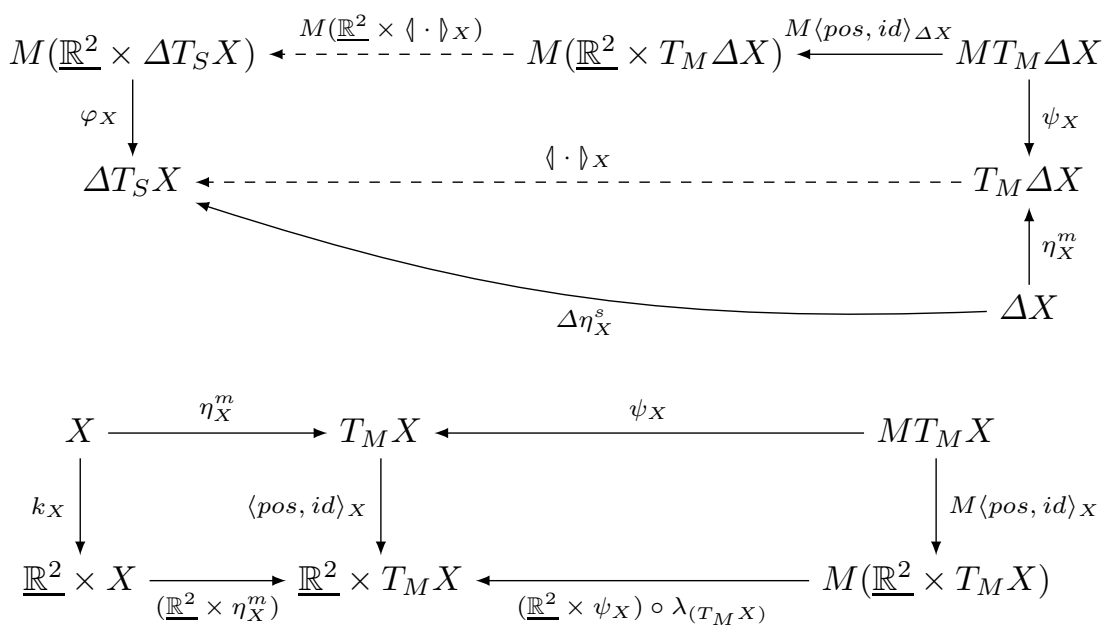

It is easy to check that the diagrams above commute also when $\left.\downarrow \cdot\right|_{X} ^{\mathrm{fl}}$ is used in place of $v \cdot\rangle_{X}$, hence $\downarrow \cdot \vartheta_{X}^{\mathrm{fl}}=\downarrow \cdot \nu_{X}$. Naturality follows by Corollary 16,

Although we have seen that the proposed $\lambda$-iteration proof principle is expressive enough for implementing a "metric" semantics for the FlatCCS (but is not limited to this) we must recall that in [6] there are other and more expressive $\lambda$-coiterative proof principles, depending on the type of the distributive law $\lambda$ at hand. Therefore, this method can be further extended by dualizing [6] in all its variants of coinductive proof principles.

\section{Conclusions}

In this paper, we have introduced Measure GSOS, an SOS specification format for continuous state probabilistic calculi, such that the induced behavioural equivalence is a congruence. As an example, we have introduced FlatCCS, a CCS-like probabilistic calculus of processes living on the Euclidean plane. 
In this format, transitions have the form $p \stackrel{\alpha}{\rightarrow} \mu$, where $\mu$ is a "measure term", i.e., an expression denoting a probability measure over sets of processes. An MGSOS specification $(\mathcal{R}, \downarrow \cdot \downarrow)$ is composed by a set $\mathcal{R}$ of GSOS-like rules, and a measure terms evaluation function $\downarrow \cdot \downarrow: T_{M} \Delta \Rightarrow \Delta T_{S}$. The rule set yields a LTS corresponding to the collective semantics of all the $\mathcal{R}$-derivable measure terms for a given process. Then, each measure term is given an "interpretation" via $\downarrow \cdot V$, and the overall probabilistic semantics is given by summing up the set of partial behaviours. MGSOS specification systems are then proved to yield an abstract GSOS distributive law of type $S\left(I d \times \Delta^{L}\right) \Rightarrow\left(\Delta T_{S}\right)^{L}$.

It is interesting to compare this format with the usual GSOS. In particular, in a transition $p \stackrel{\alpha}{\rightarrow} \mu$, the source term $p$ and the target term $\mu$ are from different languages, defined by two different syntactic monads ( $T_{S}$ and $T_{M}$ respectively). The connection between these two languages is provided by $\downarrow \cdot \lambda$, which is a kind of "distributive law" across two languages. Notably, the usual GSOS format can be seen as a special case, when $T_{S}=T_{M}$ and $\Delta$ is not present; in this case, $\downarrow \cdot \downarrow=I d$. Thus, a possible future work is to investigate a more general GSOS-like format, encompassing both MGSOS and GSOS specifications.

Another interesting future work is to extend this development to the case of continuous stochastic systems. Basically, we have to replace the probabilistic monad $\Delta$ with the stochastic monad $G$. We need to prove that this monad admits a final coalgebra, but this can be shown by adapting the proof given in 30 .

Acknowledgements. The authors wish to thank the anonymous referees for the useful comments on the preliminary version of this paper, in particular for suggesting a simpler construction of the initial algebra in the proof of Theorem 4 .

\section{References}

1. Abbott, E.A.: Flatland: A Romance of Many Dimensions. Blackwell (1884)

2. Adámek, J., Trnková, V.: Automata and Algebras in Categories, 1st edn. Kluwer Academic Publishers, Norwell (1990)

3. Bacci, G., Miculan, M.: Measurable stochastics for Brane Calculus. Theoretical Comput. Sci. 431, 117-136 (2012), doi:10.1016/j.tcs.2011.12.055

4. Barbuti, R., Maggiolo-Schettini, A., Milazzo, P., Pardini, G.: Spatial calculus of looping sequences. Electr. Notes Theor. Comput. Sci. 229(1), 21-39 (2009)

5. Barr, M.: Algebraically compact functors. Journal of Pure and Applied Algebra $82(3), 211-231$ (1992)

6. Bartels, F.: On Generalised Coinduction and Probabilistic Specification Formats: Distributive Laws in Coalgebraic Modelling. PhD thesis, CWI, Amsterdam (2004)

7. Bartels, F., Sokolova, A., de Vink, E.P.: A hierarchy of probabilistic system types. Electr. Notes Theor. Comput. Sci. 82(1) (2003)

8. Bloom, B., Istrail, S., Meyer, A.R.: Bisimulation can’t be traced. J. ACM 42(1), 232-268 (1995)

9. Brodo, L., Degano, P., Gilmore, S., Hillston, J., Priami, C.: Performance Evaluation for Global Computation. In: Priami, C. (ed.) GC 2003. LNCS, vol. 2874, pp. 229253. Springer, Heidelberg (2003) 
10. Cardelli, L., Gardner, P.: Processes in Space. In: Ferreira, F., Löwe, B., Mayordomo, E., Mendes Gomes, L. (eds.) CiE 2010. LNCS, vol. 6158, pp. 78-87. Springer, Heidelberg (2010)

11. Cardelli, L., Mardare, R.: The measurable space of stochastic processes. In: Proc. QEST, pp. 171-180. IEEE Computer Society (2010)

12. Danos, V., Desharnais, J., Laviolette, F., Panangaden, P.: Bisimulation and cocongruence for probabilistic systems. Inf. Comput. 204(4), 503-523 (2006)

13. de Vink, E.P., Rutten, J.: Bisimulation for Probabilistic Transition Systems: A Coalgebraic Approach. In: Degano, P., Gorrieri, R., Marchetti-Spaccamela, A. (eds.) ICALP 1997. LNCS, vol. 1256, pp. 460-470. Springer, Heidelberg (1997)

14. Desharnais, J., Edalat, A., Panangaden, P.: Bisimulation for labelled Markov processes. Inf. Comput. 179(2), 163-193 (2002)

15. Ding, J., Hillston, J.: Structural Analysis for Stochastic Process Algebra Models. In: Johnson, M., Pavlovic, D. (eds.) AMAST 2010. LNCS, vol. 6486, pp. 1-27. Springer, Heidelberg (2011)

16. Doberkat, E.-E.: Stochastic relations: foundations for Markov transition systems. Chapman \& Hall/CRC Studies in Informatics Series. Chapman \& Hall/CRC (2007)

17. Giry, M.: A categorical approach to probability theory. In: Banaschewski (ed.) Categorical Aspects of Topology and Analysis. Lecture Notes in Mathematics, vol. 915, pp. 68-85. Springer, Heidelberg (1982)

18. Hermanns, H., Herzog, U., Katoen, J.-P.: Process algebra for performance evaluation. Theor. Comput. Sci. 274(1-2), 43-87 (2002)

19. Hillston, J.: Process algebras for quantitative analysis. In: Proc. LICS, pp. 239-248. IEEE Computer Society (2005)

20. Klin, B., Sassone, V.: Structural Operational Semantics for Stochastic Process Calculi. In: Amadio, R.M. (ed.) FOSSACS 2008. LNCS, vol. 4962, pp. 428-442. Springer, Heidelberg (2008)

21. Kurz, A.: Logics for Coalgebras and Applications to Computer Science. PhD thesis, Ludwig-Maximilians-Universität München (2000)

22. Larsen, K.G., Skou, A.: Bisimulation through probabilistic testing. Information and Computation 94(1), 1-28 (1991)

23. Moss, L.S., Viglizzo, I.D.: Final coalgebras for functors on measurable spaces. Inf. Comput. 204(4), 610-636 (2006)

24. Panangaden, P.: Labelled Markov Processes. Imperial College Press (2009)

25. Plotkin, G.D.: A structural approach to operational semantics. DAIMI FN-19, Computer Science Department, Århus University, Århus (1981)

26. Smyth, M.B., Plotkin, G.D.: The category-theoretic solution of recursive domain equations. SIAM J. Comput. 11(4), 761-783 (1982)

27. Sokolova, A.: Probabilistic systems coalgebraically: A survey. Theoretical Comput. Sci. 412(38), 5095-5110 (2011)

28. Staton, S.: Relating coalgebraic notions of bisimulation. Logical Methods in Computer Science 7(1) (2011)

29. Turi, D., Plotkin, G.: Towards a mathematical operational semantics. In: Proc. 12th LICS Conf., pp. 280-291. IEEE Computer Society Press (1997)

30. Viglizzo, I.D.: Final Sequences and Final Coalgebras for Measurable Spaces. In: Fiadeiro, J.L., Harman, N.A., Roggenbach, M., Rutten, J. (eds.) CALCO 2005. LNCS, vol. 3629, pp. 395-407. Springer, Heidelberg (2005) 